\title{
IDEOLOGICAL CHILLOUT MIX: USO DE VISUALIDAD, ENERGÍA Y CONECTIVIDAD EN LAS APLICACIONES DE LOS SERVICIOS DE ESCUCHA DE MÚSICA EN LÍNEA
}

\author{
Verónica Soria Martínez \\ Universidad Politécnica Valencia. Dpto. Escultura. Doctoranda
}

\section{Resumen}

Por el uso que hacen de la visualidad, la energía y la conectividad, los servicios de escucha de música en línea pueden funcionar como dispositivos de uniformización social e ideológica. La visualidad de las aplicaciones, por medio de la fotografía y del diseño de la interfaz, se asocia con un énfasis en lo extramusical y en el momento del usuario. El continuo suministro de música, y los efectos que tienen sobre el comportamiento humano, hacen que se pueda hablar del concepto de energía en torno a estos servicios. Por último, la conectividad, por medio de funciones de red social incluidas en las propias aplicaciones, añade a estos servicios una dimensión social que abarca dinámicas de grupo y el uso de datos para predicciones de mercado. Este texto describe cómo estas tres vías definen cómo el usuario recibe la música que escucha y por tanto hacen de estas aplicaciones un ejemplo de interpasividad, que a su vez contribuye a una uniformización del gusto y del horizonte de posibilidades para el usuario.

\section{Palabras-clave: ESCUCHA; MERCANCÍA; TEORÍA CRÍTICA; UNIFORMIZACIÓN; INTERNET}

\section{IDEOLOGICAL CHILLOUT MIX: USE OF VISUALITY, ENERGY AND CONNECTIVITY IN THE APPLICATIONS OF ONLINE MUSIC SERVICES}

\section{Abstract}

Because of their use of visuality, energy and connectivity, online music services can work as devices for social and ideological standardization. The applications' visuality, through photography and design interface, can be related to an emphasis on the extra-musical dimension and the moment of the user. The continuous supply of music, and its effect on human behavior, allow us to speak about the concept of energy in relation to these services. Lastly, connectivity, through social networking features included in the applications themselves, adds to these services a social dimesion, encompassing group dynamics and the use of data for market predictions. This paper describes how these three ways define how the user will receive the music that he/she listens, and therefore, make of these applications an example of interpassivity, which in turn contributes to a standardization of taste and of the horizon of possibilities for the user. Keywords: HEARING; COMMODITY; CRITICAL THEORY; STANDARDIZATION;
INTERNET

Soria Martínez, Verónica. 2016. "Ideological Chillout Mix: Uso de visualidad, energía y conectividad en las aplicaciones de los servicios de escucha de música en línea". AusArt 4(1): pp-pp. 119-127 DOI: $10.1387 /$ ausart. 16688

\section{AUSART}




\section{INTRODUCCIÓN}

La llamada economía de aplicaciones y el éxito de los servicios a demanda de consumo de productos culturales han fomentado la proliferación de plataformas para la escucha de música en línea, y ello ha supuesto un cambio de paradigma en las ventas que mantienen la industria musical. Si antes existía una radio digital, como Pandora, o un servicio a la carta, como el de Spotify, especializados en música, ahora gigantes como Apple, Google o Amazon cuentan con sus propios servicios de escucha en línea. Todos estos servicios han dejado de vender música, puesto que lo que venden es el acceso a ella. Dado que estos servicios ofrecen acceso a la música como un bien mercantilizado, el propio acto de escucha se objetifica, volviéndose cuantificable y con un valor, y actúa como una mercancía. Ya no se acumulan canciones en un soporte o en un archivo, sino que se busca, se escucha, y se comparte o se "gusta". Son los músicos los que deben acumular escuchas para poder mantenerse viables económicamente, porque las escuchas se cuantifican y se monetizan. El uso de la visualidad, las corrientes de energía y la conectividad en estas aplicaciones ha modificado la manera en que pensamos en la escucha, como en el pasado lo hicieron las estaciones de radio.

\section{VISUALIDAD}

Desde que en 2015 los ingresos por escuchas en línea de canciones superaron los de las ventas de CDs, se puede hablar de un cambio de paradigma en la industria musical. En primer lugar, porque los servicios de escucha en línea usan la música y los aspectos sociales relacionados con ella como reclamo para vender el acceso a las escuchas. Para apelar a estos aspectos de tipo extramusical, se recurre al uso de la imagen y al diseño publicitario inserto en la aplicación. El uso de la publicidad en la música no es nuevo, pero sí lo es la forma que toma dentro de la aplicación. Tomemos como ejemplo Spotify. En esta plataforma, la imagen se usa para hacer publicidad del producto (las escuchas) y también del propio medio (la aplicación). En esta línea, se puede observar la importancia de la imagen publicitaria para promocionar ciertas funciones del programa, como son las listas de reproducción, que constituyen un aspecto en que la compañía está centrando sus esfuerzos para ganar usuarios. Según afirma Daniel Ek, fundador de Spotify, esto sucede porque la estrategia de la compañía tiene más que ver con el momento que con la 
propia música (Seabrook 2014). Efectivamente, las listas de reproducción se seleccionan al clicar en fotografías que ilustran el momento en que se centra cada lista: Lazy Sunday, Hipster Brunch, Morning Commute, son solo algunos ejemplos. Al distinguir unas selecciones de otras gracias a estas imágenes, se podría argüir que las canciones que se promocionan en las listas más escuchadas tienen parámetros tan similares que es más fácil diferenciarlas por lo que estan haciendo los personajes en las fotografías.

\subsection{La imagen publicitaria en LAS PLATAFORMAS dE ESCUCHA EN LÍNEA}

Gracias a la publicidad explícita, como las canciones y otros productos que se anuncian, se crean unos intereses de tipo económico que solo pueden materializarse si se continúa usando el medio. Por eso, se da otra publicidad de tipo implícita, la del propio medio, que se centra en gran parte en los datos generados por los propios usuarios. Por ello, existe en estas aplicaciones una mediación en el usuario a través de la imagen, puesto que las fotografías relacionan la música con la fabricación de formas de vida atractivas, generando un proceso publicitario que afecta los deseos y valores del oyente y modifica su capacidad de acción. Este aspecto se ve reforzado a través de lo que dicen las canciones, que relacionan a los artistas y a los oyentes con una determinada visión del mundo. Por último, estos mensajes publicitarios se repiten en los perfiles de los consumidores al compartir las canciones que los contienen (en Spotify todo lo que se escucha queda registrado y se anuncia a los contactos del usuario), por lo que la conectividad activa esta mediación en cada interacción con la aplicación.

Además, existe un componente de interpasividad en el aspecto visual de Spotify. Žižek y Pfaller definen la obra de arte interpasiva como aquella que se observa a sí misma (Pfaller 2003; Žižek 2003). Un ejemplo de ellas serían las series de TV con risa enlatada, puesto que la comedia se ríe de sí misma mientras el espectador observa. De igual modo, la fotografías de la aplicación indican lo que se debe sentir al escuchar las canciones: observamos a los modelos estudiar, relajarse o reir en las listas cuyos títulos incluyen palabras como "Focus", "Lazy", "Happy". Son un ejemplo de interpasividad como "entretenimiento delegado" (Pfaller 2003) a la aplicación. 


\subsection{USO INTUITIVO GRACIAS A LA IMAgEN Y EL DISEÑO}

En la escucha de música mediante aplicaciones no hay manipulación directa de objetos, sólo una interfaz plana diseñada para ser manejada de manera intuitiva y que a menudo se encuentra en pantallas táctiles. Cualquiera puede usar la aplicación, no se requiere conocer "cómo funciona". Para dar una idea del cambio que se ha producido de manera casi desapercibida, en los 80 no se permitía a los niños poner música, porque no podían manipular el tocadiscos o el ordenador. Sin embargo, un bebé de hoy día, antes del año de edad ya sabe hacer fotos y poner música en un smartphone. Además, estas aplicaciones son baratas - o gratis - para el usuario y no necesitan ningún espacio de almacenamiento. Por todo esto, se han convertido en la solución eficaz para el consumo cotidiano de la música, que se relega al plano de fondo durante la realización de otras actividades. No obstante, cuantas más facilidades de uso representa una tecnología, más configuraciones predeterminadas conlleva, limitando las elecciones que puede realizar el usuario, y reduciendo el horizonte de posibilidades que puede escoger (Morozov 2013). En el caso de las plataformas de escucha online, además de activarse fácilmente, una primera activación genera un uso continuado durante horas, ya que la aplicación continúa seleccionando música de manera ininterrumpida y ofrece más recomendaciones.

\section{ENERGÍA}

Los servicios de escucha en línea pueden funcionar "a la carta" o generando selecciones de escuchas. Las listas y recomendaciones son generadas por sistemas de IA sofisticados, en combinación con especialistas humanos (como en el caso del trabajo realizado por The Genome Project para Pandora) o barridos de contenido existente en la web (como en el de The Echo Nest para Spotify).

Este tipo de escucha recuerda a una corriente de energía que se retroalimenta. No requiere ningún tipo de participación, puesto que, una vez activada, el suministro puede durar horas. Es más, se paga por el acceso ininterrumpido, sin publicidad, que se ofrece a los socios. Así, estas aplicaciones están diseñadas para poder usar música como sonido de fondo, para acompañar al usuario y distraer su pensamiento mientras hace otras cosas. 
Al hacer esto, otorgamos responsabilidad a la aplicación, delegando en ella el placer del descubrimiento y la selección de la música acorde con el momento. De nuevo volviendo al concepto de interpasividad, Žižek (2003) pone como ejemplo el aparato de video que graba en cintas los programas que no vemos, transformando nuestra actividad pasiva-receptiva consciente en un frenesí de actividad sin conciencia. El problema con la escucha automática se agudiza porque seguimos ahí, escuchando sin escuchar. Aunque no prestemos atención, el oído no filtra los mensajes que le llegan casi de manera desapercibida.

Por otro lado, si cada escucha se monetiza, es de suponer que la decisión no será del todo arbitraria. En el caso específico de Spotify, los tres grandes sellos (Sony, Warner y Universal) y un grupo de sellos independiente (Merlin) se reparten entre un 15 y un $18 \%$ de sus acciones. A estas compañías les interesa que se escuchen sus productos. En este sentido, en 2014 ya se detectó en Pandora una alteración de los algoritmos en favor de grupos pertenecientes al catálogo de Merlin a cambio de un descuento en la tasa que la compañía paga a los sellos. Pero aunque estos algoritmos no estuvieran modificados para favorecer determinados intereses, su funcionamiento se basa en que todo lo que se selecciona es similar a lo primero que introdujo el usuario en el buscador, lo que ya constituye un ciclo repetitivo en torno a los mismos parámetros.

Se puede hablar de energía, no solamente por la manera en que funcionan estos servicios, sino también por la influencia que ejercen en el estado anímico del oyente, pues la música se utiliza, por parte del usuario, como una "tecnología del yo", que ayuda a regular sus emociones en el momento, y además, a largo plazo, se crea una identificación con determinados valores e ideas que se asocian a la música (DeNora 2000, 46-74), puesto que los valores atribuidos a la música que escoge el oyente, se entrelazan con la percepción de sí mismo. Además, la música puede ayudar con la concentración, quitar el aburrimiento de las tareas caseras, mejorar el rendimiento durante el ejercicio físico, relajarse, ir a dormir e incluso ayudar a ambientar una velada romántica (Levitin 2007) y crear fácilmente una 'banda sonora' que sea fiel reflejo de nuestra personalidad, y que al mismo tiempo sea capaz de aportarnos confort y motivación". Cuando este poder que tiene la música se utiliza de manera que los oyentes se ven expuestos de manera desapercibida, como por ejemplo, en comercios, negocios y oficinas, la capacidad de elección o acción del individuo puede verse afectada. Estudios demuestran cómo oir una música de club en una tienda de ropa atrae a un tipo de clientes y alejará otros, incitando con ellos un tipo de ventas sobre otras (DeNora 2000, 
145). Los servicios de escucha en línea cuentan con diseños específicos que sirven tanto para estimular el consumo de los clientes de los comercios como para mantener el humor de los trabajadores de una empresa. Por ejemplo, Spotify cuenta con una modalidad de suscripción para negocios y empresas que permite realizar listas de reproducción que se adaptan a las necesidades del entorno y al momento del día, cada día de la semana. Así, se convierte en una herramienta que en potencia puede ayudar a definir el comportamiento de amplios grupos humanos.

Este tipo de funcionamiento conduce a una uniformización de la escucha, puesto que se favorecen canciones que sirven para la decoración del tiempo y la generación de imágenes positivas. Por otro lado, considerando que "nos gusta música que es parecida a lo que nos gustó anteriormente, pero no demasiado similar" (Levitin 2007), los servicios de escucha en línea que generan escuchas automáticas garantizan un producto que produce continuamente diferentes versiones de lo mismo que ya se ha escuchado.

\section{CONECTIVIDAD}

Los servicios de escucha en línea cuentan con un componente de red social. Por ejemplo, Spotify permite seguir a otros usuarios y compartir con ellos lo que se escucha en cada momento. Pero además, existe una relación de reciprocidad con la red social Facebook. La conectividad entre los usuarios facilita la recopilación de datos sobre su identidad, gustos, relaciones, pertenencias e intereses, lo que permite su agrupación en sectores destinatarios de publicidad. De esta manera, Spotify puede garantizar el éxito de sus listas y recomendaciones porque se basan en datos generados por el usuario a través de tres vías: los propios hábitos de escucha del usuario, la información introducida por este en la plataforma social de Spotify y, por último, a través de la información depositada en Facebook.

Los datos generados se supervisan regularmente y se venden a otras empresas. Gracias a ellos, Spotify sabe a qué hora del día se escuchan ciertas canciones, y gracias a los smartphones, también la situación geográfica de los usuarios. De esta manera, los programadores pueden inferir lo que aquellos están haciendo probablemente, como "estudiar, hacer ejercicio, conducir 
al trabajo" (Seabrook 2014). Esto a su vez les ayuda a generar las próximas recomendaciones.

Por otro lado, la dimensión social de la escucha, en otras palabras, lo que recomiendan y esuchan los amigos, ha sido destacada tanto por especialistas en psicología de la escucha (Levitin 2007) como por el experto en descubrimientos musicales de Spotify (Whitman 2013). Pero de esta manera, lo que se pone de manifiesto es otro elemento uniformizador, ya que se recomienda lo más popular en ciertos círculos. Es decir, se incita a la escucha de lo que ya se escucha, sintonizando con la presión de grupo y con la necesidad de inclusión del individuo, a la manera de "los artículos más leídos" en los periódicos en línea.

Por último, se perpetúa el círculo repetitivo de afirmación del gusto, otorgando una renovada vigencia a la frase de Adorno acerca de la radio comercial, que afirma que la selección musical "se reproduce en un círculo vicioso: lo más conocido es lo que más éxito tiene; por lo que se vuelve siempre a reproducir y se vuelve todavía más popular" (Adorno [1956] 2003, 22). Experimentos hechos públicos por Spotify y otras aplicaciones revelan cómo a partir de los datos de usuario se pueden generar predicciones en cuanto a artistas o canciones de mayor éxito en el futuro, lo que se ha denominado como el efecto Shazam. Como contrapartida, los productores y agentes buscarán, para asegurar el éxito, talentos que continúen con las mismas tendencias que han obtenido una popularidad reciente.

\section{CONCLUSIÓN}

Los servicios de escucha en línea favorecen la generación de ciclos de repetición a través de la visualidad de su interfaz, la energía que las mantiene funcionando y la conectividad gracias a la cual interactúan sus usuarios. Por medio de esto, se da lugar a una nueva versión de la mediación capitalista, produciéndose en un sistema cerrado, o círculo repetitivo. En este círculo, se distinguen tres niveles: el uso individual de la música, el uso social de pequeño grupo (como puede ser el poner música en una fiesta), y en último lugar, el uso en grandes grupos sociales (como las modalidades de Spotify para el lugar de trabajo y para los comercios). 
En primer lugar, la visualidad, a través de la imagen, articula los deseos en el lenguaje publicitario, y a través del diseño, facilita los hábitos de escucha automática de los usuarios. En segundo lugar, la energía, en forma del estado anímico del usuario, y en forma de algoritmos que mantienen funcionando la aplicación, genera datos que se estudian para realizar las recomendaciones en que se basan estos servicios y customizar la publicidad. Por último, la conectividad garantiza la interactuación entre usuarios y la perpetuación del servicio, así como la propagación de la publicidad, explícita, a través de los anuncios comerciales, como implícita, a través de las tendencias generadas por los usuarios.

Esto confiere al servicio una dimensión expandida de lo extramusical que se relaciona con rasgos con los que el usuario se identifica. A través del servicio, su aspecto visual, su funcionamiento y su conectividad, se promueven determinadas formas de ser, que se identifican con formas de ver el mundo. Al reducirse las posibilidades al historial de uso propio o al de las personas a las que el sistema engloba en un mismo nicho de mercado, disminuye la variedad de perfiles posibles y, con estos, las formas de ver el mundo. En última instancia, se trata de un círculo en el que, bajo la premisa de que el usuario escoge lo que quiere escuchar, se forma una secuencia única, convenciendo al usuario de que lo auténticamente desea es lo mismo que se le está ofreciendo, en cada nueva versión.

\section{Referencias}

Adorno, Theodor W. (1956) 2003. Dissonanzen: Musik in der verwalteten Welt. Frankfurt: Suhrkamp

Areni, Charles S. and David Kim. 1993. "The influence of background music on shopping behavior: classical versus top-forty music in a wine store". Advances in Consumer Research 20: $336-40$

Bernasek, Anna \& D.T. Mongan. 2015. "Big data is coming for your purchase history - to charge you more money". The Guardian, 29 mayo. http://www.theguardian.com/commentisfree/2015/may/29/big-data-purchase-history-charge-you-more-money

DeNora, Tia. 2000. Music and everyday life. Cambridge: Cambridge University

Elberse, Anita. 2013. Blockbusters: Hit-making, risk-taking, and the big business of entertainment. New York: Henry Holt and Co.

Guattari, Pierre-Félix. (1992) 1996. Caosmosis. Traducción, Irene Agoff. Buenos Aires: Manantial 
Levitin, David. 2007. "Life soundtracks: the uses of music in everyday life: Report prepared for the exclusive use of Philips Consumer Electronics B.V., Eindhoven, The Netherlands" http:// www.russballard.com/rbv7-workshop/physics/Unit\%203\%20Vibrations/LifeSoundtracks.pdf

Macias, Dave. 2012. "Making dollars: Clearing up Spotify payment confusion", Hypebot.com: Music, Technology, The new music business (blog), 26 noviembre. http://hypebot.com/ hypebot/2012/11/clearing-up-spotify-payment-confusion.html

Morozov, Evgeny. 2013. "Is smart making us dumb?" Wall Street Journal, 23 febrero. http:// www.wsj.com/articles/SB10001424127887324503204578318462215991802

Peoples, Glenn. 2015. "The digital world is flat: Streaming gains negated by CD slumps in many global markets". Billboard, 27 febrero. http://www.billboard.com/articles/business/6487413/ global-music-revenue-report-2015-streaming-gains-physical-sales

Pfaller, Robert. 2003. "Backup of little gestures of disappearance: Interpassivity and the Theory of Ritual". Journal of European Psycoanalysis: Humanities, Philosophy, Psychotherapies 16 (Winter-Spring).www.psychomedia.it/jep/number16/pfaller.htm

Prévost, Edwin. 2010. "Improvisación libre en la música y capitalismo: La resistencia a la autoridad y el culto por el cientificismo y la celebridad". En Ruido y capitalismo, Mattin [Mattin Artiach Oráa] \& Anthony lles, eds., 42-63. Donostia: Arteleku Audiolab. www.arteleku.net/ audiolab/ruido capitalismo.pdf

Sanneh, Kelefa. 2013. "Blockbuster" The New Yorker, 2 diciembre www.newyorker.com/magazine/2013/12/02/blockbluster

Seabrook, John. 2014. "Spotify: Friend or foe?" The New Yorker, 24 noviembre. http://www. newyorker.com/magazine/2014/11/24/revenue-streams

Taylor, Astra. 2014. The people's platform: Taking back power and culture in the digital age. New York: Metropolitan Books

Thompson, Derek. 2014. "The Shazam effect". The Atlantic, December issue. http://www.theatlantic.com/magazine/archive/2014/12/the-shazam-effect/382237/

Whitman, Brian. 2013. "How music recommendation works — and doesn't work". Notes.variogr. am [web personal del autor]. http://notes.variogr.am/post/37675885491/howmusic-Recommendation-works-and-doesnt-work

Žižek, Slavoj. 2003. "Will you laugh for me, please". Lacam.com. http://lacan.com/zizeklaugh. htm 